\title{
LUPUS RETINOPATHY: A SILENT MANIFESTATION IN A PATIENT WITH RECENT DIAGNOSIS OF SYSTEMIC LUPUS ERYTHEMATOSUS
}

Caroline Pamponet da Fonseca Oliveira ${ }^{1, \star}$, Camila Pamponet da Fonseca Oliveira² ${ }^{2}$ Isabelle Santos Alves ${ }^{2}$, Junior Apaestegui Coriat $^{1}$, Gabriela Cruz de Oliveira ${ }^{1}$, Bruno Meireles Brito de Souza ${ }^{3}$, Bárbara Seabra Carneiro ${ }^{1}$

1.Hospital Universitário Getúlio Vargas, Manaus (AM), Brazil. 2.Universidade Federal do Amazonas, Manaus (AM), Brazil. 3.Secretaria Estadual de Saúde do Estado do Amazonas, Manaus (AM), Brazil.

*Corresponding author: carolinepamponetfo@gmail.com

\section{BACKGROUND}

Ophthalmological impairments are a common finding in systemic lupus erythematosus (SLE), affecting about one third of patients. Lupus retinopathy is a poorly recognized manifestation of the disease. Mild and visually nonthreatening symptoms are frequent. However, retinopathy can be an indicator of disease activity and be associated with more severe disorders, resulting in a worst prognosis. It may be caused by two main mechanisms: vasculitis or retinal vessel thrombosis induced by antiphospholipid antibodies.

\section{CASE REPORT}

C.C.S.C., 17 years old, female, presenting photosensitivity, malar rash, low-grade fever, oral ulcer on palate, alopecia and arthralgia for 5 months. Laboratory tests showed positive double helix anti-dsDNA, low C3 and C4, erythrocyte sedimentation rate of $40 \mathrm{~mm}$ and antinuclear factor (ANF) Hep2 1:640 homogeneous nuclear pattern. Evolved with lower limb edema +++/4, foamy urine, oliguria, elevated blood pressure levels and $24 \mathrm{~h}$ proteinuria of $1,400 \mathrm{mg}$. The patient closed the diagnosis of SLE, adding 26 points to the 2019 ACR/EULAR classification criteria. Prednisone $60 \mathrm{mg}$ and hydroxychloroquine $400 \mathrm{mg}$ were started for treatment. She was referred to ophthalmology according to protocol, despite the absence of visual complaints. Then she was diagnosed with acute lupus retinopathy. She had negative venereal disease research laboratory (VDRL) and viral serology. On retinography, hemorrhage was observed in a temporal candle flame to the optic disc on the right in a papillomacular bundle (Fig. 1); in left eye, hemorrhage in candle flame was noted in the lower arch; in addition, bilaterally inferior cotton wool exudates inferior to the macula were present (Fig. 2). In fluorescein angiography, there were two areas of hypofluorescence by block, temporal to the optic disc, in a papillomacular bundle in the right eye (Fig. 3). To control disease activity, the patient underwent pulse therapy with methylprednisolone and cyclophosphamide.

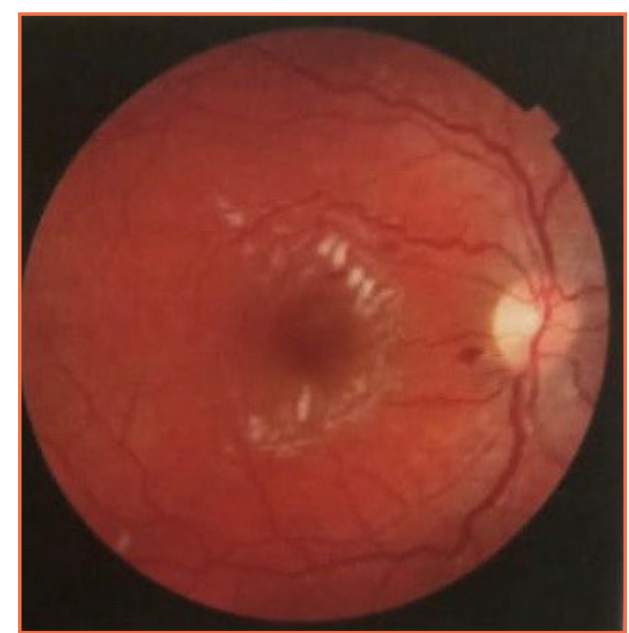

Figure 1. Right eye retinography. 


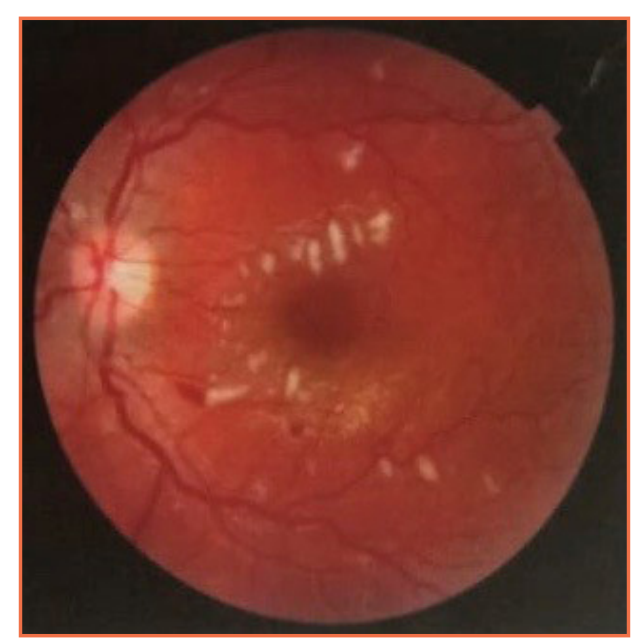

Figure 2. Left eye retinography.

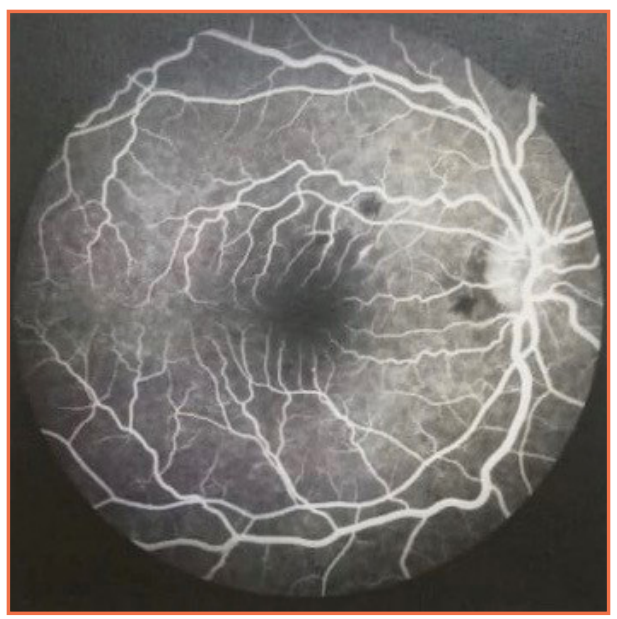

Figure 3. Right eye fluorescein angiography.

\section{CONCLUSION}

Ocular manifestations in SLE can occur at the beginning of the disease or during its evolution, affecting any part of the visual system. However, in clinical practice, many patients with active disease are not examined by a retinal specialist, and visual symptoms are generally not documented during medical evaluation. 